\title{
Clinical Study \\ Cardiopulmonary Response to Exercise Testing in People with Chronic Stroke: A Retrospective Study
}

\author{
Sandra A. Billinger, ${ }^{1}$ Jordan M. Taylor, ${ }^{1}$ and Barbara M. Quaney ${ }^{2}$ \\ ${ }^{1}$ Department of Physical Therapy and Rehabilitation Science, University of Kansas Medical Center, 3901 Rainbow Boulevard, \\ Mail Stop 2002, Kansas City, KS 66160, USA \\ ${ }^{2}$ Landon Center on Aging, University of Kansas Medical Center, 3901 Rainbow Boulevard, Mail Stop 1005, Kansas City, \\ KS 66160, USA
}

Correspondence should be addressed to Sandra A. Billinger, sbillinger@kumc.edu

Received 5 May 2011; Revised 7 July 2011; Accepted 14 July 2011

Academic Editor: Gillian Mead

Copyright ( $\odot 2012$ Sandra A. Billinger et al. This is an open access article distributed under the Creative Commons Attribution License, which permits unrestricted use, distribution, and reproduction in any medium, provided the original work is properly cited.

\begin{abstract}
Background and Purpose. This study investigated the cardiopulmonary response and safety of exercise testing at peak effort in people during the chronic stage of stroke recovery. Methods. This retrospective study examined data from 62 individuals with chronic stroke (males: 32; mean (SD); age: (12.0) yr) participating in an exercise test. Results. Both males and females had low cardiorespiratory fitness levels. No significant differences were found between gender for peak $\mathrm{HR}(P=0.27)$, or $\mathrm{VO}_{2}$ peak $(P=0.29)$. Males demonstrated higher values for minute ventilation, tidal volume, and respiratory exchange ratio. No major adverse events were observed in the exercise tests conducted. Discussion and Conclusion. There are differences between gender that may play a role in exercise testing performance and should be considered when developing exercise programs. The low $\mathrm{VO}_{2}$ peak of this cohort of chronic stroke survivors suggests the need for participation in exercise interventions.
\end{abstract}

\section{Introduction}

Recovery from stroke is challenging due to impaired neuromuscular control, decreased functional mobility, balance deficits, and reduced cardiorespiratory (CR) fitness $[1,2]$. Emerging evidence suggests exercise training in the poststroke population can facilitate improvements in the cardiovascular, respiratory, and neuromuscular systems [3, 4]. For a variety of reasons, stroke survivors are not routinely prescribed aerobic exercise during stroke rehabilitation [5], which likely exacerbates their decline in cardiorespiratory fitness. Research studies have demonstrated not only improvements in $\mathrm{VO}_{2}$ peak after exercise interventions [3, $4,6,7]$ but also physical function (e.g., Timed Up and Go), walking $[8,9]$, and psychological well being $[8,10]$. Yet, many clinicians do not employ aerobic exercise interventions, perhaps because of the limited amount of research that has identified appropriate screening protocols and optimal dosing of aerobic exercise for this population $[11,12]$.
Furthermore, the current literature available to healthcare professionals regarding the safety and feasibility of exercise testing in people with chronic stroke (i.e., $\geq 6$ months [13]) has been limited by mostly smaller data sets ( $<35$ participants with chronic stroke).

CR fitness is important for performing daily activities and mobility. It has been reported that $\mathrm{VO}_{2}$ peak values below $20 \mathrm{~mL} * \mathrm{~kg}^{-1} * \mathrm{~min}^{-1}$ is associated with limited physical function for instrumental activities of daily living [14, 15]. After stroke, women, when compared to men, need more assistance for activities of daily living and walking [16]. In stroke rehabilitation, women may need to participate in aerobic exercise training to increase endurance for daily activities. While it is known that CR fitness is reduced after stroke, the majority of the studies to date have enrolled a higher proportion of men. In fact, most studies describing CR fitness after stroke have less than 25\% [7, 13, 17, 18] or no women [19] enrolled. It is important to understand the cardiopulmonary response to exercise testing in both 
men and women so that the physiologic and hemodynamic responses obtained from an exercise test can be used to appropriately guide exercise prescription [2].

While most studies report $\mathrm{VO}_{2}$ peak and $\mathrm{HR}$ as outcomes from an exercise test in the stroke population, little information is available regarding other outcome measures involving the pulmonary system such as minute ventilation (VE), tidal volume, or ventilatory efficiency of carbon dioxide $\left(\mathrm{VE} / \mathrm{VCO}_{2}\right)$ in this clinical population. In an early study of stroke survivors with left-sided hemiplegia, respiratory function was compromised and may have "contributed to fatigue that limits physical activity" [20].

Therefore, the aims of this retrospective study were to characterize and provide a comprehensive description of cardiopulmonary responses during an incremental exercise test in chronic stroke survivors (i.e., $\geq 6$ months) and report on the range of responses to exercise testing between gender. We hypothesized that men, after stroke, would have higher values for CR fitness and pulmonary function when compared to women. We also wanted to determine which cardiovascular, pulmonary, and functional outcomes would predict CR fitness using peak oxygen consumption $\left(\mathrm{VO}_{2}\right.$ peak). Finally, we sought to determine if individuals in the chronic stage of stroke can safely participate in exercise testing using the data set available.

\section{Methods}

For the present work, data were gathered and analyzed from exercise testing results that were conducted in the University of Kansas, General Clinical Research Center (GCRC) during the time period from 2005 to 2009 . These studies included individuals with chronic stroke who participated in peak effort exercise test. The admission criteria into the previous studies have been described elsewhere [21-23]. Briefly, inclusion criteria were (1) single ischemic stroke at least 6 months prior to enrollment, (2) ability complete sit to stand transfer, (3) independent with ambulation 30 feet with or without an orthotic or assistive device, and (4) Mini Mental Status Exam score of $\geq 24[21,22]$, and both studies excluded individuals with cardiac limitations that were absolute contraindications for exercise testing [24]. The modalities used for the exercise tests were either a cycle ergometer (Lode BV, Zernikepark 16, 9747 AN Groningen, Netherlands) (Lode BV, Groningen, Netherlands), or total body recumbent stepper (NuStep, Inc, 51111 Venture Dr., Ann Arbor, MI 48108) (NuStep, Ann Arbor, MI). Both exercise test protocols used 2-minute incremental stages. In our previous work, we reported a strong relationship between the cycle ergometer and total body recumbent stepper testing protocols for $\mathrm{VO}_{2}$ peak $(r=$ $0.91, P<0.001)$ in people after stroke [22].

A brief description for the exercise testing protocols is provided. Individuals were instructed to abstain from consuming food and caffeine 3 hours prior to the scheduled test. Calibration procedures were performed on the metabolic cart (ParvoMedics, 8152 South 1715 East, Sandy, UT 84093) (ParvoMedics True One 2400, Sandy UT) according to the manufacturer specifications. An exercise physiologist familiarized each participant at the time of the visit with the exercise equipment, the mouthpiece, noseclip, exercise testing protocol, and procedures and explained the Borg rating of perceived exertion (RPE) scale. Participants were attached to a 12-lead electrocardiograph (ECG) to continuously monitor heart rate and rhythm. A 2-way, nonrebreathing valve, headgear, mouthpiece, and nose clip were worn by the participants. Blood pressure and RPE were acquired during the last 30 seconds of each stage. Expired gases were collected continuously, and oxygen uptake and carbon dioxide production was averaged at 15-second intervals. The exercise test was terminated if the participant reached volitional exhaustion or met absolute test termination criteria according to ACSM guidelines [24].

Sixty-two individuals with chronic stroke who participated in an exercise test in the GCRC were identified for inclusion into the study. Exclusion criteria consisted of missing or incomplete data. The study procedures were followed in accordance with the institutional guidelines and were approved by the University's Human Subjects Committee. Participant characteristics are listed in Table 1.

We examined the cardiopulmonary indices at peak effort for the entire group and across gender. Due to the variability in age range ( 26 to 81 years of age) of the study population, we performed a subanalysis of the data. We separated our data into age groups by decade as listed in the ACSM's Guidelines for Exercise Testing and Prescription [24]. This allowed for characterization of aerobic fitness levels across gender and age to describe our participant's percentile rank for maximal aerobic power.

Most individuals in this dataset had enrolled in an intervention study. To avoid any confounds associated with exercise interventions, we assessed the cardiopulmonary response to exercise testing data only from baseline exercise tests.

\section{Data Analysis}

The arithmetic mean and standard deviation were used for descriptive statistics. Primary outcome measures and body composition were tested for normality using a KolmogorovSmirnov 1 sample test. A univariate general linear model was used to examine gender differences for cardiopulmonary parameters. To account for differences in peak HR, medications that affect HR (beta blockers and $\mathrm{Ca}+$ channel blockers) were used as covariates in the statistical analyses. To determine which parameters could have the greatest influence on $\mathrm{VO}_{2}$ peak $\left(\mathrm{mL} * \mathrm{~kg}^{-1} * \mathrm{~min}^{-1}\right)$ for the cohort, a stepwise linear regression model was performed. In order to examine 5 factors in the regression analysis, we would need at least 50 people to be included in the sample. Therefore, the independent variables included in the regression analysis were peak VE $\left(\mathrm{L} * \mathrm{~min}^{-1}\right)$, peak $\mathrm{HR}$, age, gender, and lower extremity Fugl-Meyer. Pearson correlation coefficients were used to examine the relationship between $\mathrm{VO}_{2}$ peak and those significant factors in the regression model. All analyses were conducted using SPSS statistical software (SPSS, Inc, 233 S Wacker Dr., Chicago, Ill 60606) (SPSS, v 17) with the alpha level $<0.05$. 
TABle 1: Participant demographics.

\begin{tabular}{|c|c|c|c|}
\hline Characteristics $n=62$ & Group mean $(\mathrm{SD})^{\ddagger}$ & Males & Females \\
\hline Sex: male & & 32 & 30 \\
\hline Age (years) & $62.0(12.0)$ & $68.0(10.5)$ & $55.0(11.9)^{*}$ \\
\hline Height (m) & $1.7(0.09)$ & $1.8(0.05)$ & $1.6(0.05)^{*}$ \\
\hline Weight (kg) & $87.7(18.0)$ & $92.5(14.8)$ & $82.5(19.9)$ \\
\hline Body mass index & $29.3(5.4)$ & $28.5(4.2)$ & $30.2(6.5)$ \\
\hline \multicolumn{4}{|l|}{ Race/ethnicity } \\
\hline African American & 11 & 2 & 9 \\
\hline Caucasian & 46 & 28 & 18 \\
\hline Hispanic & 4 & 2 & 2 \\
\hline Native American & 1 & 0 & 1 \\
\hline \multicolumn{4}{|l|}{ Stroke characteristics } \\
\hline Time (months) after stroke & $61.0(50.0)$ & $62.9(35.9)$ & $57.9(61.2)$ \\
\hline Right side weakness & 31 & 19 & 12 \\
\hline Bilateral deficits & 1 & 1 & 0 \\
\hline LE Fugl-Meyer score & $23.3(7.2)$ & $24.7(6.3)$ & $21.8(7.8)$ \\
\hline \multicolumn{4}{|l|}{ Comorbidities for cardiac risk } \\
\hline Type 2 diabetes mellitus & 21 & 12 & 9 \\
\hline Current smoker & 0 & 0 & 0 \\
\hline Overweight (BMI 25.0-29.9) [24] & 25 & $27.5(1.5)$ & $27.6(1.6)$ \\
\hline Obese (BMI 30.0 to $\geq 40$ ) [24] & 27 & $33.0(1.7)$ & $34.2(4.6)$ \\
\hline \multicolumn{4}{|l|}{ Medications: } \\
\hline high blood pressure & 40 & 20 & 20 \\
\hline beta blockers & 21 & 9 & 12 \\
\hline calcium channel blockers & 15 & 5 & 10 \\
\hline ACE inhibitors & 30 & 16 & 14 \\
\hline hyperlipidemia & 17 & 9 & 8 \\
\hline
\end{tabular}

${ }^{\ddagger}$ Data are " $n$ " participants of 62 unless indicated by group mean (SD).

\section{Results}

4.1. Cardiopulmonary Response to Exercise Testing. Data for the primary cardiovascular and pulmonary outcomes and body composition were normally distributed $(P$-values $>$ $0.310)$. Males were older, taller, and weighed more than their female counterparts (Table 1). Despite these differences in physical parameters, no significant differences were found between genders for peak HR even when controlling for medications $(P=0.27)$. Females were younger and their age-predicted HR max (APHRM) calculated by 220 minus age was significantly higher than males. However, peak HR at the end of the exercise test was lower than their male counterparts despite being younger. Furthermore, the number of males $(n=28)$ and females $(n=29)$ reporting the use of a beta blockade was similar. $\mathrm{VO}_{2}$ peak $\left(\mathrm{mL} * \mathrm{~kg}^{-1} * \mathrm{~min}^{-1}\right)$ $(P=0.29)$ and lower extremity function using the FuglMeyer score $(P=0.11)$ were also not significantly different. Pulmonary function for minute ventilation $\left(\mathrm{L} * \mathrm{~min}^{-1}\right)$ and tidal volume (L) were significantly higher in males while respiratory rate was not different between genders. We also report that males exercised beyond their anaerobic threshold with respiratory exchange ratio (RER) significantly higher. However, their perceived exertion at peak effortwas similar. Cardiopulmonary exercise parameters from the initial exercise test $(n=62)$ are listed in Table 2.

$\mathrm{VO}_{2}$ peak is reported as the index for CR fitness and in the poststroke population, and these values have been reported well below age-matched, sedentary controls [18]. Therefore, according to the normative data provided in ACSM's Guidelines for Exercise Testing and Prescription [24], we divided the cohort into age groups by decade. All stroke survivors but one had $\mathrm{VO}_{2}$ peak values below the first percentile, which classifies their CR fitness as very poor. Only one person in the 20-29-year-old age range had a $\mathrm{VO}_{2}$ peak in the 10th percentile (poor category). Furthermore, only 10 of the 62 individuals had $\mathrm{VO}_{2}$ peak values above $20 \mathrm{~mL} *$ $\mathrm{kg}^{-1} * \min ^{-1}$. This is important to consider since $\mathrm{VO}_{2}$ peak values below $20 \mathrm{~mL} * \mathrm{~kg}^{-1} * \mathrm{~min}^{-1}$ has associated with limited physical function for instrumental activities of daily living. Table 3 lists $\mathrm{VO}_{2}$ peak values across age groups.

4.2. Regression Model. A stepwise linear regression was performed to examine which cardiopulmonary measures and stroke outcomes contributed to $\mathrm{VO}_{2}$ peak. Our results showed that minute ventilation (VE) was the most predictive and significant measure of $\mathrm{VO}_{2}$ peak for chronic stroke survivors 
TABLE 2: Cardiopulmonary response at peak effort in chronic stroke.

\begin{tabular}{|c|c|c|c|}
\hline Characteristics ( $n=62$ exercise tests) & Group & Males & Females \\
\hline Relative peak $\mathrm{VO}_{2}\left(\mathrm{~mL} * \mathrm{~kg}^{-1} * \mathrm{~min}^{-1}\right)$ & $15.6(5.42)$ & $16.3(3.6)$ & $14.8(6.8)$ \\
\hline $\mathrm{VO}_{2}$ peak $\left(\mathrm{L} * \min ^{-1}\right)$ & $1.3(0.42)$ & $1.5(0.4)$ & $1.2(0.4)^{*}$ \\
\hline HR reached at peak effort (bpm) & $127.4(28.8)$ & $131.4(29.6)$ & $123.2(27.8)$ \\
\hline$\%$ of age-predicted HR Max (APHRM) & & $86 \%$ & $75 \%$ \\
\hline Age-predicted HR Max (bpm) (220 minus age) & $158.3(12.8)$ & $152.3(10.5)$ & $164.7(11.9)^{*}$ \\
\hline RER & $1.06(0.1)$ & $1.10(0.1)$ & $1.02(0.1)^{*}$ \\
\hline $\mathrm{VE}\left(\mathrm{L} * \min ^{-1}\right)$ & $51.3(18.7)$ & $61.2(18.0)$ & $40.7(12.9)^{*}$ \\
\hline Tidal volume (L) & $1.6(0.5)$ & $1.8(0.4)$ & $1.3(0.5)^{*}$ \\
\hline Breathing frequency (breaths $* \min ^{-1}$ ) & $33.0(8.5)$ & $34.0(8.6)$ & $32.0(8.4)$ \\
\hline Ventilatory equivalent for $\mathrm{CO}_{2}\left(\mathrm{VE} / \mathrm{VCO}_{2}\right)$ & $36.5(6.4)$ & $37.2(6.0)$ & $35.7(6.8)$ \\
\hline Rating of perceived exertion & $16.0(2.0)$ & $16.5(1.8)$ & $16.0(2.2)$ \\
\hline
\end{tabular}

$\mathrm{VO}_{2}$ peak, peak oxygen uptake; HR: heart rate; RER: respiratory exchange ratio; Peak VE, minute ventilation, and $\mathrm{CO}_{2}$ : carbon dioxide. All values are expressed as mean (standard deviation). ${ }^{*}$ denotes significance between males and females $(P<0.01)$.

TABLE 3: Age comparison of cardiopulmonary exercise test parameters and LE function.

\begin{tabular}{|c|c|c|c|c|}
\hline Age group (years) & Gender $(n=)$ & LE Fugl Meyer score & $\mathrm{VO}_{2}\left(\mathrm{~mL} * \mathrm{~kg}^{-1} * \mathrm{~min}^{-1}\right)$ & Percentile rank* \\
\hline \multirow{2}{*}{$20-29$} & $\mathrm{~F}(1)$ & 34 & 28.7 & 10th \\
\hline & $\mathrm{M}(0)$ & 一 & - & \\
\hline \multirow{2}{*}{$30-39$} & $\mathrm{~F}(1)$ & 13 & 14.9 & Below 1st \\
\hline & M (1) & 31 & 21.5 & Below 1st \\
\hline \multirow{2}{*}{$40-49$} & $\mathrm{~F}(8)$ & $19.4(8.3)$ & $12.1(3.8)$ & Below 1st \\
\hline & $\mathrm{M}(1)$ & 27 & 24.6 & Below 1st \\
\hline \multirow{2}{*}{$50-59$} & $\mathrm{~F}(9)$ & $23.4(8.7)$ & $16.9(9.0)$ & Below 1st \\
\hline & $\mathrm{M}(1)$ & 22 & 19.2 & Below 1st \\
\hline \multirow{2}{*}{$60-69$} & $\mathrm{~F}(7)$ & $23.9(4.9)$ & $15.1(6.6)$ & Below 1st \\
\hline & M (14) & $23.6(7.2)$ & $15.2(3.2)$ & Below 1st \\
\hline \multirow{2}{*}{$70-79$} & $\mathrm{~F}(4)$ & $18.8(7.8)$ & $11.6(2.4)$ & Below 1st \\
\hline & M (12) & $25.3(5.6)$ & $15.9(3.6)$ & Below 1st \\
\hline \multirow{2}{*}{$80-89$} & $\mathrm{~F}(0)$ & - & - & \multirow{2}{*}{ No normative data } \\
\hline & M (3) & $21.8(7.5)$ & $17.4(1.9)$ & \\
\hline
\end{tabular}

${ }^{*}$ Normative data for percentile values for maximal aerobic power from American College of Sports Medicine's Exercise Testing Guidelines and Prescription. LE: Lower extremity.

$\left(R^{2}=0.37, P<0.001\right)$ (Figure 1$)$ although adding LE FuglMeyer scores markedly improved the model prediction $R^{2}=$ $0.51, P<0.001)$.

Adding peak HR only slightly improved the overall model $\left(R^{2}=0.54, P<0.001\right)$. Age and gender did not improve the model. Pearson correlation coefficients suggest a significant relationship between lower extremity Fugl-Meyer scores and $\mathrm{VO}_{2}$ peak (Figure $2 ; r=0.57, P<0.001$ ).

4.3. Safety of Exercise Testing in Chronic Stroke. Major adverse events were defined according to the American College of Sports Medicine Guidelines for Exercise Testing and Prescription [24] (e.g., moderately severe angina, drop in systolic blood pressure of $>10 \mathrm{~mm} \mathrm{Hg}$ from baseline blood pressure, ST elevation, sustained ventricular tachycardia). In our data set, no major adverse events (i.e., death, heart attack) were observed. However, one cardiac complication (ST segment depression $>2 \mathrm{~mm}$ ) was reported. At the onset of this event, the exercise test was terminated and ST segment depression returned to baseline within 1 minute. The individual's cardiologist was notified, but hospitalization was not required. Another exercise test was terminated early because the participant experienced increased discomfort with the mouthpiece. Therefore, the mouthpiece was removed, and the test was terminated. One individual reported feeling dizzy after the exercise test was completed, although no cardiac symptoms were recorded (i.e., normal blood pressure and $\mathrm{HR}$ response), and the dizziness subsided after two minutes. All other exercise tests were terminated due to volitional fatigue.

\section{Discussion}

The primary aims of this retrospective study were to describe the cardiopulmonary response to an incremental exercise test and determine whether gender differences existed and had 


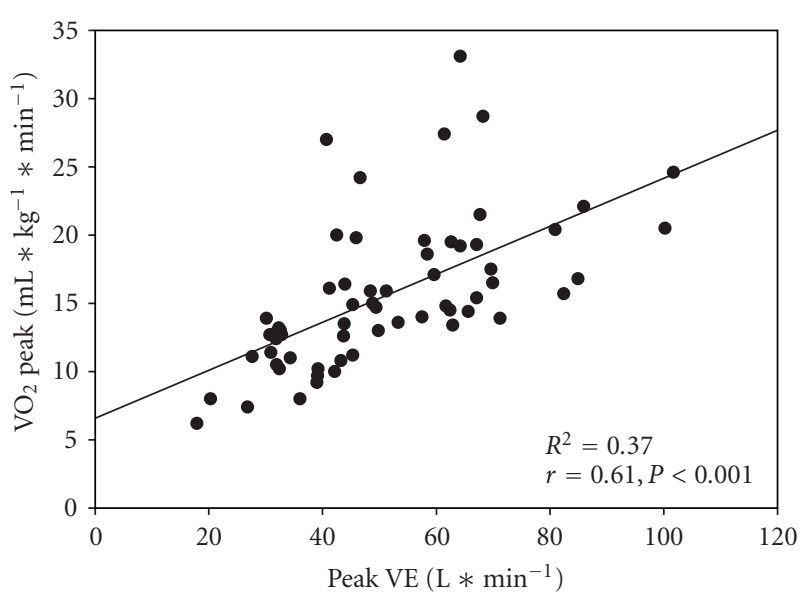

FIGURE 1: Scatterplot with a regression line overlay to demonstrate the relationship between $\mathrm{VO}_{2}$ peak and peak minute ventilation (VE).

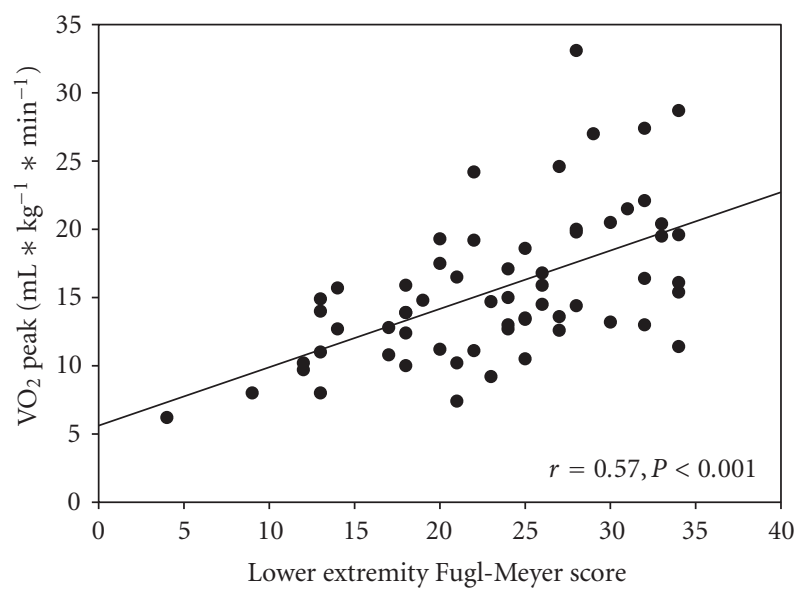

FIGURE 2: Scatterplot with a regression line overlay to demonstrate the relationship between $\mathrm{VO}_{2}$ peak and lower extremity function using the Fugl-Meyer.

an effect on exercise testing performance. We sought to investigate which of the cardiopulmonary and functional measures would predict $\mathrm{VO}_{2}$ peak, which may help emphasize the need for aerobic training programs in stroke rehabilitation. Finally, we wanted to assess the safety of exer-cise testing to peak effort in people with chronic stroke.

\section{Cardiopulmonary Response to Exercise Testing}

$\mathrm{VO}_{2}$ peak has been consistently shown to be a useful measure of functional capacity. Specifically, two recent studies [14, 15] reported that $\mathrm{VO}_{2}$ peak values below $20 \mathrm{~mL} * \mathrm{~kg}^{-1} * \mathrm{~min}^{-1}$ is associated with limited performance in activities of daily living. In our cohort of chronic stroke survivors, approximately $84 \%$ had $\mathrm{VO}_{2}$ peak values below this functional capacity. Further, in our study, the mean value for $\mathrm{VO}_{2}$ peak $(\mathrm{mL}$
$* \mathrm{~kg}^{-1} * \mathrm{~min}^{-1}$ ) in both genders after stroke was 55\% and $43 \%$ lower in females and males, respectively, than those reported for healthy sedentary counterparts [25]. This severe reduction in $\mathrm{VO}_{2}$ peak could have functional implications for independent living, return to work and community participation in people after stoke [18]. Since thepulmonary system is also essential during exercise but poorly described in people after stroke, we sought to examine these outcome variables in addition to the cardiac system. We found group mean VE values comparable to those reported by two previous studies in stroke survivors $[18,26]$ but less than those reported for healthy adults [26, 27]. We found only one other report by Tomczak and colleagues that examined tidal volume and breathing frequency for pulmonary function during exercise testing after stroke [26], and our results are similar in findings. Ventilatory equivalent for carbon dioxide $\left(\mathrm{VE} / \mathrm{VCO}_{2}\right)$ reflects ventilatory efficiency, and values below 30 are considered normal [27]. The findings listed in Table 3 demonstrate an abnormal, high response with the group mean for $\mathrm{VE} / \mathrm{VCO}_{2}$ ratio at 36.5. The higher $\mathrm{VE} / \mathrm{VCO}_{2}$ ratio response, along with decreased $\mathrm{VE}$ and tidal volume, may be indicative of a high physiologic dead space fraction in the lungs [28, 29]. Rehabilitation professionals need to consider aerobic exercise interventions and inspiratory muscle training targeted towards increasing pulmonary function after stroke.

\section{Gender Differences}

Females had significantly lower values for absolute peak oxygen uptake $\left(\mathrm{L} * \mathrm{~min}^{-1}\right)$, RER, VE, and tidal volume than males. However, $\mathrm{VO}_{2}$ peak when normalized to body weight $\left(\mathrm{mL} * \mathrm{~kg}^{-1} * \mathrm{~min}^{-1}\right)$ was lower than the males but not significantly different. Despite females being younger than their male counterparts, peak HR for females was also lower than males (123.2 (27.8) versus 131.4 (29.6), resp.) but not statistically different when we controlled for cardiac medications $(P=0.27)$. It is possible that with a larger sample size, we would find statistically significant differences between males and females for the cardiovascular outcomes. However, an article by MacKay-Lyons and colleagues [18] briefly mentioned gender differences in their data set, but they had a smaller sample size and an unequal distribution of males $(n=22)$ and females $(n=7)$. In their study, they reported that males had significantly higher $\mathrm{VO}_{2}$ peak values than females early after stroke. We are unclear to the reason for this finding since our female group was significantly younger (55.0 (11.9) versus 68.0 (10.5)) than the males. Our available data does not lend insight to other cardiopulmonary or physical parameters (earlier onset of fatigue) that may have affected their exercise performance. Additionally, we also did not have information regarding other measures that can be clinically meaningful during submaximal efforts such as the oxygen uptake efficiency slope or the $\mathrm{VO}_{2}$ /Work rate slope. Since we performed a retrospective analysis, the data should be interpreted with caution. We acknowledge that future prospective studies examining gender differences should have a sample that is age matched 
to determine the role of gender on these cardiovascular and pulmonary parameters.

When we compared the effect of gender on pulmonary outcomes at peak effort from the exercise test, we found that males had larger minute ventilation (VE) and tidal volume, but respiratory rates were similar to females. However, both groups were below normative values for ventilation at peak exercise effort [27]. VE and tidal volume for males in this study were $63 \%$ and $66 \%$ of normal values but had respiratory rates similar to healthy adults in these age ranges ( $94 \%$ of normal). Females' values were also lower with VE and tidal volume at 59\% and 68\% of the normative data, respectively. Females also showed near normal respiratory rates at $89 \%$ of normative values. The response of the respiratory system in people after stroke both at submaximal and maximal effort may provide insight towards potential explanations regarding shortness of breath during activity and fatigue experienced after stroke. As shown in our data, early fatigue onset may partially be due to respiratory insufficiency through decreased lung volumes $[20,30]$. Impaired breathing mechanics could be related to the extent of motor impairment (e.g., paresis of the hemidiaphragm, intercostal and abdominal muscles) and these individuals could benefit from inspiratory muscle training to increase strength and excursion [31,32]. Given the paucity of data on gender-based differences in the cardiopulmonary response of stroke survivors on exercise testing, we describe a rather diverse group of chronic stroke survivors to help define the gender effects on peak exercise capacity.

\section{Regression Model}

According to the stepwise linear regression, VE was the most predictive measure of $\mathrm{VO}_{2}$ peak. We also report that the Lower Extremity Fugl-Meyer (LEFM) score was a significant contributor in $\mathrm{VO}_{2}$ peak as suggested by the step-wise regression model. In fact, as demonstrated in Table 3, those with higher LEFM scores also had higher $\mathrm{VO}_{2}$ peak values. Work done by Macko and colleagues suggests that the hemiparetic leg may not be the primary deciding factor in test termination [13]. While we cannot discount lower extremity function of the affected leg (Fugl-Meyer score), there are other underlying factors such as reduced (1) lean tissue [33], (2) blood flow [34-36], and (3) work performance [37] in the hemiparetic leg that may contribute to early test termination in people after stroke.

However, the mechanisms that may be fundamental to the severe reductions in $\mathrm{VO}_{2}$ peak observed here cannot be acquired from this retrospective data set. We also acknowledge that different exercise modalities may elicit differences in $\mathrm{VO}_{2}$ peak, $\mathrm{HR}$, and exercise testing performance [24]. However, our previous work comparing the exercise testing protocols used for cycle ergometer and the recumbent stepper demonstrated a strong association for $\mathrm{VO}_{2}$ peak $(r=$ $0.91, P<0.001)$ and HR $(r=0.89, P<0.001)$ [22], and, in the present study, we had a similar distribution of males and females that used the two types of exercise equipment (bike, recumbent stepper) to avoid bias of exercise modality.

\section{Exercise Testing Safety}

The results of our study provide strong evidence that both baseline and repeated maximal effort exercise tests during the chronic stages of stroke recovery are safe and welltolerated by this high cardiac risk clinical population. We report a high percentage $(99 \%)$ of participants that were able to complete the symptom-limited exercise test without a cardiac complication. Previous work demonstrated the safety and feasibility of exercise testing in the chronic stroke population but the sample sizes were small $[13,21,22]$. The data from this study supports the work of others that exercise testing is safe when conducted with the proper level of screening and monitoring $[4,37,38]$.

This information regarding exercise testing safety is important and relevant for those professionals working with people after stroke. Our data suggest that males and females after stroke have very poor CR fitness. The American Heart Association's Scientific Statement, Physical Activity and Exercise Recommendations for Stroke Survivors and The Best Practice Guidance for the Development of Exercise after Stroke Services in Community Settings [39], states the importance of incorporating aerobic exercise into stroke rehabilitation [11] and beyond to improve the health and well being of stroke survivors [39]. Further, the current literature has demonstrated aerobic exercise to be beneficial for improving CR fitness $[6,14,36,40]$, quality of life [4], gait and sixminute walk test $[4,40]$, and functional performance [14]. On the basis of this study, it appears that aerobic exercise interventions to improve CR fitness should be incorporated into stroke rehabilitation programs.

Since the literature suggests that most individuals after stroke present with existing coronary artery disease (CAD) [30] along with other cardiac risk factors, exercise testing can be a useful screening tool for cardiovascular complications prior to beginning an exercise intervention. Despite these risk factors, a high percentage $(99 \%)$ of individuals completed the exercise testing without minor cardiac complications, and we report no serious adverse events. Exercise testing in the post-stroke population can provide useful information related to cardiovascular and pulmonary performance, exercise prescription parameters, and reassurance to the individual after stoke that exercise is safe and beneficial.

It should be noted that these results may not be representative of the stroke population. For instance, the individuals in this dataset volunteered for exercise studies and may be more confident, as they were willing to participate in peak effort exercise testing. We tried to provide a comprehensive representation of cardiopulmonary parameters obtained during exercise testing. While the exercise testing protocols routinely assess blood pressure, some charts did not have the final values listed at peak effort, or it was unclear if the value was obtained at peak. Therefore, blood pressure was not included. Current medications were selfreported by the participant on the day of the exercise test to 
the exercise physiologist. It is possible that there may be some inaccuracies in reporting, which may have unintentionally confounded the data. However, we made every effort to obtain the recent medication list from the participant or family member.

\section{Conclusion}

Rehabilitation professionals should encourage people after stroke to participate in exercise interventions to improve cardiorespiratory health. We report that gender differences do exist in cardiopulmonary variables obtained through exercise testing with HR response below the APHR max, defined as 220 minus age suggesting that other methods (e.g., rating of perceived exertion) may be more reliable. These findings for gender and poor CR fitness should be considered by rehabilitation professionals when developing exercise prescription parameters in rehabilitation and community settings to optimize the cardiorespiratory benefits associated with exercise. Regardless of age, individuals after stroke have severe and significant reductions in peak oxygen uptake. However, for stroke survivors who wish to return to independent living, work, or community activities, their functional capacity and participation may be limited due to CR fitness. In conclusion, our findings suggest that exercise testing is safe in people with chronic stroke and may be beneficial in guiding exercise prescription.

\section{Acknowledgments}

This work was supported by the American Heart Association (NDSG 0530208N, BQ); National Institutes of Health, NICHD K01 HD47148-05 (BQ) and Grant Number M01 RR023940 from the National Center for Research Resources (NCRR), a component of the National Institutes of Health (NIH) (BQ, SAB). The authors wish to thank Craig Harms, $\mathrm{PhD}$, FACSM for his helpful comments in manuscript preparation, and Gabe Harter for technical assistance with manuscript preparation.

\section{References}

[1] M. J. MacKay-Lyons and J. Hewlett, "Exercise capacity and cardiovascular adaptations to aerobic training early after stroke," Topics in Stroke Rehabilitation, vol. 12, no. 1, pp. 3144, 2005.

[2] K. Palmer-McLean and K. Harbst, Acsm's Exercise Management for Persons with Chronic Diseases and Disabilities, Human Kinetics Books, Champaign, Ill, USA, 3rd edition, 2009.

[3] R. F. Macko, F. M. Ivey, L. W. Forrester et al., "Treadmill exercise rehabilitation improves ambulatory function and cardiovascular fitness in patients with chronic stroke: a randomized, controlled trial," Stroke, vol. 36, no. 10, pp. 22062211, 2005.

[4] A. Tang, K. M. Sibley, S. G. Thomas et al., "Effects of an aerobic exercise program on aerobic capacity, spatiotemporal gait parameters, and functional capacity in subacute stroke," Neurorehabilitation and Neural Repair, vol. 23, no. 4, pp. 398406, 2009.
[5] M. J. MacKay-Lyons and L. Makrides, "Cardiovascular stress during a contemporary stroke rehabilitation program: is the intensity adequate to induce a training effect?" Archives of Physical Medicine and Rehabilitation, vol. 83, no. 10, pp. 13781383, 2002.

[6] J. H. Rimmer, A. E. Rauworth, E. C. Wang, T. L. Nicola, and B. Hill, "A preliminary study to examine the effects of aerobic and therapeutic (nonaerobic) exercise on cardiorespiratory fitness and coronary risk reduction in stroke survivors," Archives of Physical Medicine and Rehabilitation, vol. 90, no. 3, pp. 407412, 2009.

[7] R. F. Macko, G. V. Smith, C. L. Dobrovolny, J. D. Sorkin, A. P. Goldberg, and K. H. Silver, "Treadmill training improves fitness reserve in chronic stroke patients," Archives of Physical Medicine and Rehabilitation, vol. 82, no. 7, pp. 879-884, 2001.

[8] G. E. Mead, C. A. Greig, I. Cunningham et al., "Stroke: a randomized trial of exercise or relaxation," Journal of the American Geriatrics Society, vol. 55, no. 6, pp. 892-899, 2007.

[9] D. H. Saunders, C. A. Greig, G. E. Mead, and A. Young, "Physical fitness training for stroke patients," Cochrane Database of Systematic Reviews, no. 4, Article ID CD003316, 2009.

[10] R. Harrington, G. Taylor, S. Hollinghurst, M. Reed, H. Kay, and V. A. Wood, "A community-based exercise and education scheme for stroke survivors: a randomized controlled trial and economic evaluation," Clinical Rehabilitation, vol. 24, no. 1, pp. 3-15, 2010.

[11] N. F. Gordon, M. Gulanick, F. Costa et al., "Physical activity and exercise recommendations for stroke survivors: an American heart association scientific statement from the council on clinical cardiology, subcommittee on exercise, cardiac rehabilitation, and prevention; the council on cardiovascular nursing; the council on nutrition, physical activity, and metabolism; and the stroke council," Circulation, vol. 109, no. 16, pp. 20312041, 2004.

[12] C. Meek, A. Pollock, J. Potter, and P. Langhorne, "A systematic review of exercise trials post stroke," Clinical Rehabilitation, vol. 17, no. 1, pp. 6-13, 2003.

[13] R. F. Macko, L. I. Katzel, A. Yataco et al., "Low-velocity graded treadmill stress testing in hemiparetic stroke patients," Stroke, vol. 28, no. 5, pp. 988-992, 1997.

[14] A. Letombe, C. Cornille, H. Delahaye et al., "Early post-stroke physical conditioning in hemiplegic patients: a preliminary study," Annals of Physical and Rehabilitation Medicine, vol. 53, no. 10, pp. 632-642, 2010.

[15] M. E. Cress and M. Meyer, "Maximal voluntary and functional performance levels needed for independence in adults aged 65 to 97 years," Physical Therapy, vol. 83, no. 1, pp. 37-48, 2003.

[16] M. Kelly-Hayes, A. Beiser, C. S. Kase, A. Scaramucci, R. B. D'Agostino, and P. A. Wolf, "The influence of gender and age on disability following ischemic stroke: the Framingham study," Journal of Stroke and Cerebrovascular Diseases, vol. 12, no. 3, pp. 119-126, 2003.

[17] C. L. Dobrovolny, F. M. Ivey, M. A. Rogers, J. D. Sorkin, and R. F. Macko, "Reliability of treadmill exercise testing in older patients with chronic hemiparetic stroke," Archives of Physical Medicine and Rehabilitation, vol. 84, no. 9, pp. 1308-1312, 2003.

[18] M. J. MacKay-Lyons and L. Makrides, "Exercise capacity early after stroke," Archives of Physical Medicine and Rehabilitation, vol. 83, no. 12, pp. 1697-1702, 2002.

[19] R. F. Macko, C. A. DeSouza, L. D. Tretter et al., "Treadmill aerobic exercise training reduces the energy expenditure and cardiovascular demands of hemiparetic gait in chronic stroke patients: a preliminary report," Stroke, vol. 28, no. 2, pp. 326330, 1997. 
[20] A. Haas, H. A. Rusk, H. Pelosof, and J. R. Adam, "Respiratory function in hemiplegic patients," Archives of Physical Medicine and Rehabilitation, vol. 48, no. 4, pp. 174-179, 1967.

[21] B. M. Quaney, L. A. Boyd, J. M. McDowd et al., "Aerobic exercise improves cognition and motor function poststroke," Neurorehabilitation and Neural Repair, vol. 23, no. 9, pp. 879885, 2009.

[22] S. A. Billinger, B. Y. Tseng, and P. M. Kluding, "Modified totalbody recumbent stepper exercise test for assessing peak oxygen consumption in people with chronic stroke," Physical Therapy, vol. 88, no. 10, pp. 1188-1195, 2008.

[23] S. A. Billinger, L. X. Guo, P. S. Pohl, and P. M. Kluding, "Single limb exercise: pilot study of physiological and functional responses to forced use of the hemiparetic lower extremity," Topics in Stroke Rehabilitation, vol. 17, no. 2, pp. 128-139, 2010.

[24] ACSM, Guidelines for Exercise Testing and Prescription, Lippincott Williams \& Wilkins, Philadelphia, Pa, USA, 2009.

[25] K. F. Hossack and R. A. Bruce, "Maximal cardiac function in sedentary normal men and women: comparison of age-related changes," Journal of Applied Physiology Respiratory Environmental and Exercise Physiology, vol. 53, no. 4, pp. 799-804, 1982.

[26] C. R. Tomczak, A. Jelani, R. G. Haennel, M. J. Haykowsky, R. Welsh, and P. J. Manns, "Cardiac reserve and pulmonary gas exchange kinetics in patients with stroke," Stroke, vol. 39, no. 11, pp. 3102-3106, 2008.

[27] S. P. Blackie, M. S. Fairbarn, N. G. McElvaney, P. G. Wilcox, N. J. Morrison, and R. L. Pardy, "Normal values and ranges for ventilation and breathing pattern at maximal exercise," Chest, vol. 100, no. 1, pp. 136-142, 1991.

[28] G. J. Balady, R. Arena, K. Sietsema et al., "Clinician's guide to cardiopulmonary exercise testing in adults: a scientific statement from the American heart association," Circulation, vol. 122, no. 2, pp. 191-225, 2010.

[29] J. Myers, R. Arena, R. B. Oliveira et al., "The lowest VE/ $/ \mathrm{VCO}_{2}$ ratio during exercise as a predictor of outcomes in patients with heart failure," Journal of Cardiac Failure, vol. 15, no. 9, pp. 756-762, 2009.

[30] E. J. Roth, "Heart disease in patients with stroke: incidence, impact, and implications for rehabilitation. Part 1: classification and prevalence," Archives of Physical Medicine and Rehabilitation, vol. 74, no. 7, pp. 752-760, 1993.

[31] S. T. Sutbeyaz, F. Koseoglu, L. Inan, and O. Coskun, "Respiratory muscle training improves cardiopulmonary function and exercise tolerance in subjects with subacute stroke: a randomized controlled trial," Clinical Rehabilitation, vol. 24, no. 3, pp. 240-250, 2010.

[32] R. R. Britto, N. R. Rezende, K. C. Marinho, J. L. Torres, V. F. Parreira, and L. F. Teixeira-Salmela, "Inspiratory muscular training in chronic stroke survivors: a randomized controlled trial," Archives of Physical Medicine and Rehabilitation, vol. 92, no. 2, pp. 184-190, 2011.

[33] A. S. Ryan, C. L. Dobrovolny, G. V. Smith, K. H. Silver, and R. F. Macko, "Hemiparetic muscle atrophy and increased intramuscular fat in stroke patients," Archives of Physical Medicine and Rehabilitation, vol. 83, no. 12, pp. 1703-1707, 2002.

[34] S. A. Billinger, B. J. Gajewski, L. X. Guo, and P. M. Kluding, "Single limb exercise induces femoral artery remodeling and improves blood flow in the hemiparetic leg poststroke," Stroke, vol. 40, no. 9, pp. 3086-3090, 2009.

[35] F. M. Ivey, A. W. Gardner, C. L. Dobrovolny, and R. F. Macko, "Unilateral impairment of leg blood flow in chronic stroke patients," Cerebrovascular Diseases, vol. 18, no. 4, pp. 283-289, 2004.
[36] F. M. Ivey, C. E. Hafer-Macko, A. S. Ryan, and R. F. MacKo, "Impaired leg vasodilatory function after stroke: adaptations with treadmill exercise training," Stroke, vol. 41, no. 12, pp. 2913-2917, 2010.

[37] A. Tang, K. M. Sibley, S. G. Thomas, W. E. McIlroy, and D. Brooks, "Maximal exercise test results in subacute stroke," Archives of Physical Medicine and Rehabilitation, vol. 87, no. 8, pp. 1100-1105, 2006.

[38] J. S. Yates, S. Studenski, S. Gollub et al., "Bicycle ergometry in subacute-stroke survivors: feasibility, safety, and exercise performance," Journal of Aging and Physical Activity, vol. 12, no. 1, pp. 64-74, 2004.

[39] "Best practice guidance for the development of exercise after stroke services in community settings," Press Release, 2010.

[40] K. Michael, A. P. Goldberg, M. S. Treuth, J. Beans, P. Normandt, and R. F. Macko, "Progressive adaptive physical activity in stroke improves balance, gait, and fitness: preliminary results," Topics in Stroke Rehabilitation, vol. 16, no. 2, pp. 133-139, 2009. 


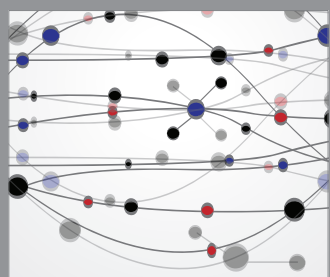

The Scientific World Journal




Gastroenterology

Research and Practice
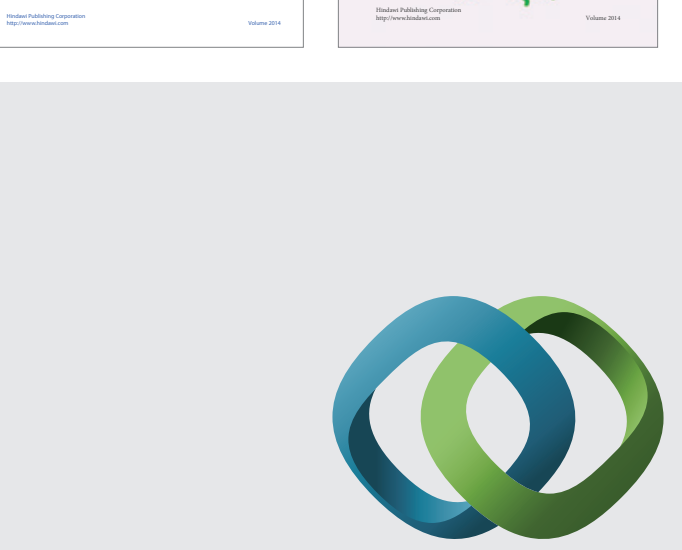

\section{Hindawi}

Submit your manuscripts at

http://www.hindawi.com
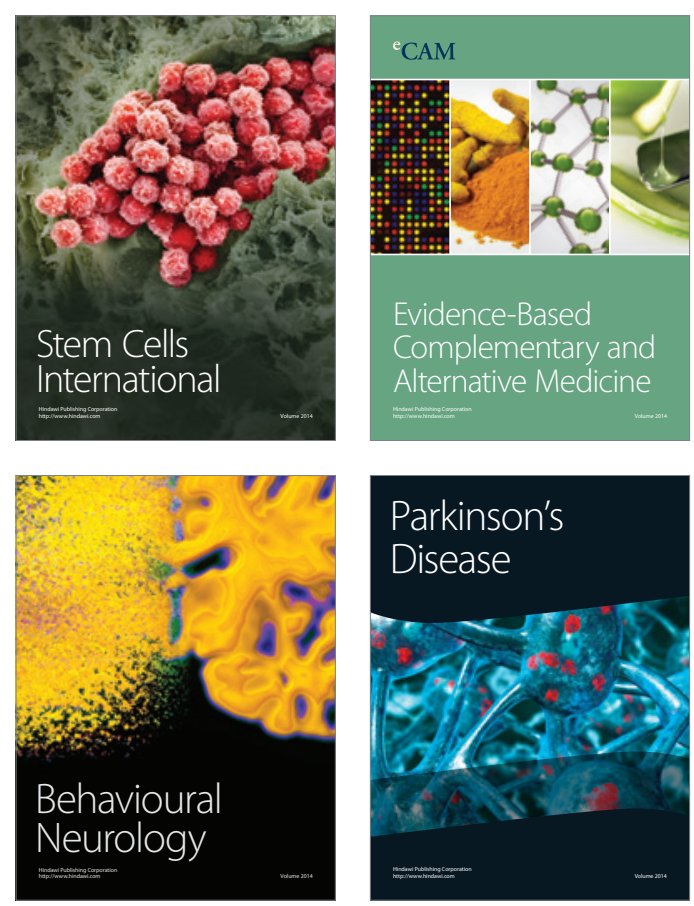

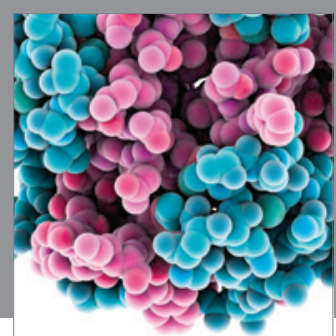

Journal of
Diabetes Research

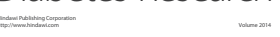

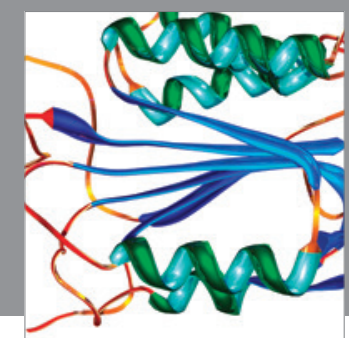

Disease Markers
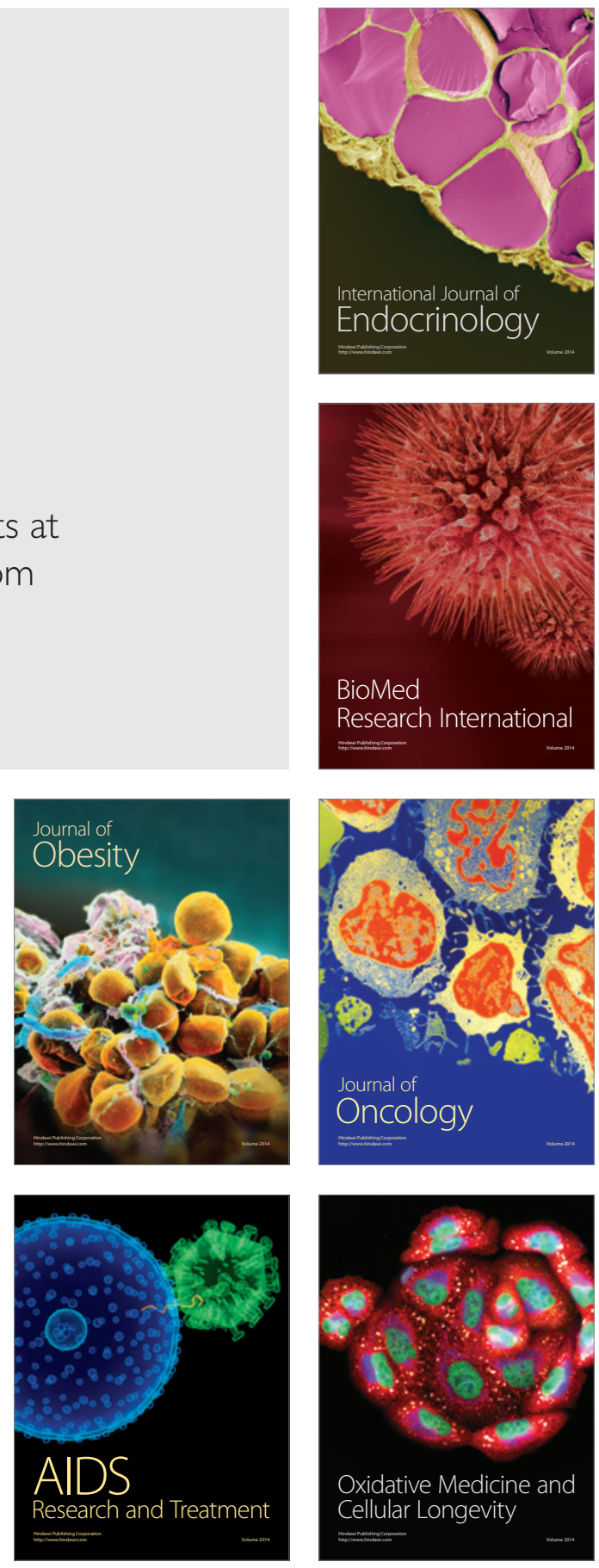